\title{
Numerical Modeling of Silicon Photodiodes for High-Accuracy Applications Part III: Interpolating and Extrapolating Internal Quantum-Efficiency Calibrations
}

\section{Jon Geist}

National Institute of Standards and Technology, Gaithersburg, MD 20899

and
A. M. Robinson and C. R. James
University of Alberta, Alberta, Canada T6G 2G7

The semiconductor device modeling program PC-1D and the programs that support its use in high-accuracy modeling of photodiodes, all of which were described in Part I of this series of papers, are used to simulate the interpolation of high-accuracy internal quantum-efficiency calibrations in the spcctral region between $450 \mathrm{~nm}$ and $850 \mathrm{~nm}$. Convenient interpolation formulae that depend only upon wavelength are derived. Uncertainty spectra for a number of sources of error are also derived. The formulae are normalized to experimental internal-quantum efficiency calibrations in the 440 to 470 $\mathrm{nm}$ spectral region and at $860 \mathrm{~nm}$ and arc used to intcrpolate the calibration values between these wavelcngths. The results of the interpolations are compared with experimental calibration data that arc available at a few wavelengths between 440 and $860 \mathrm{~nm}$. The disagreement between the interpolated and measured internal quantum-efficiency data is never worse than 0.0003 .

Key words: extrapolation; internal quantum-efficiency; interpolation; photodiode; silicon; visible.

Accepted: May 29, 1991

\section{Introduction}

Part II of this series of papers suggested that the Hamamatsu ${ }^{1} 1337$ type photodiode might be conveniently used for interpolating or extrapolating highaccuracy quantum efficiencies in the 400 to $900 \mathrm{~nm}$ spectral region. The idea of quantum-efficiency extrapolation was implicit in the first detailed description of how a silicon photodiode could be used (in this case in conjunction with a thermal detector having a spectrally flat responsivity) to realize a scale of absolute spectral responsivity [1], and interpolation was explicitly considered in reference [2]. Beyond these two publications, however, little progress has been reported in this area until recently.

\footnotetext{
${ }^{1}$ Certain commercial equipment, instruments, or materials are identified in this paper to specify adequately the experimental procedure. Such identification does not imply recommendation or cndorsement by the National Institute of Standards and Technology, nor does it imply that the materials or equipment identified are neccssarily the best available for the purpose.
}

Hoyt et al. [3] (using early results of the work described here) used three Hamamatsu 1337 type photodiodes to extrapolate a quantum-efficiency calibration obtained at $441.6 \mathrm{~nm}$ with a UDT QED 100 radiometer to $633.0 \mathrm{~nm}$, and reported an average difference of $0.04 \%$ compared to independent measurements based on their electrically calibrated cryogenic cavity radiometer. Even though $0.04 \%$ is quite good by conventional radiometric standards, it was a factor of two larger than the combined uncertainty estimate for the two measurement techniques being compared, and further study was proposed by Hoyt et al. More recently, Zalewski and Hoyt [4] have reported a more direct test of the accuracy of the use of the Hamamatsu 1337 type photodiode to extrapolate quantum-efficiency values. The results of this test agreed to within the combined estimated uncertainty, and two errors were discovered in the earlier work. When these 
errors were corrected, agreement within the estimated uncertainty of the earlier intercomparison was obtained.

The purpose of Part III of this series of papers is to develop formulae and uncertainty estimates for interpolating and extrapolating internal quantumefficiency calibrations with Hamamatsu 1337 photodiodes. It will be shown that this type of photodiode has characteristics that suit it particularly well for this task over the 440 to $860 \mathrm{~nm}$ spectral region. The remainder of Part III is organized as follows: Section 2 investigates the errors associated with the use of relations that are only approximate for the 1337 type photodiode, but which simplify interpolation and extrapolation of the internal quantum deficiency (one minus the internal quantum efficiency) for this type of photodiode. Section 3 investigates the various sources of error associated with the actual calculation of internal quantum-deficiency spectra using the semiconductor-device modeling program PC-1D and the support programs described in Part I. Finally, section 4 compares the results obtained here with experimental measurements and demonstrates agreement within 0.0003 .

\section{Errors Associated with the Quantum- Deficiency Approximations}

For the purposes of extrapolating and interpolating the internal quantum efficiency of Hamamatsu 1337 type photodiodes, the internal quantum deficiency can be approximated by a function $\delta\left(\lambda, N_{\mathrm{ss}}\right.$, $S, \tau_{\mathrm{r}}$ ) of wavelength $\lambda$ and of three recombinationrelated variables: $N_{\text {ss }}$ the charge number density stored in the front-surface oxide, $S$ the surface recombination velocity at the oxide-silicon interface, and $\tau_{\mathrm{r}}$ the minority-carrier lifetime in the rear region.

For silicon photodiodes, $\delta\left(\lambda, N_{\mathrm{ss}}, S, \tau_{\mathrm{r}}\right)$ can be approximated by a function that has properties that make it particularly useful for extrapolating and interpolating quantum-deficiency measurements. The approximate equation for $\delta\left(\lambda, N_{\mathrm{ss}}, S, \tau_{\mathrm{r}}\right)$ is

$$
\begin{aligned}
& \delta\left(\lambda, N_{\mathrm{ss}}, S, \tau_{\mathrm{r}}\right)=K_{\mathrm{f}}\left(N_{\mathrm{ss}}, S\right) \delta_{\mathrm{f}}(\lambda) \\
& +K_{\mathrm{r}}\left(\tau_{\mathrm{r}}\right) \delta_{\mathrm{r}}(\lambda)
\end{aligned}
$$

where

$$
\delta_{r}(\lambda)=0,
$$

for $\lambda$ less than some wavelength $\lambda_{0}$.
To see how this is useful for interpolating quantum-deficiency values, suppose that $\delta\left(\lambda_{t}, N_{\mathrm{ss}}, S, \tau_{\mathrm{r}}\right)$ has been measured at wavelength $\lambda_{\mathrm{f}}<\lambda_{0}$, and $\delta\left(\lambda_{\mathrm{r}}\right.$, $N_{\mathrm{ss}}, S, \tau_{\mathrm{r}}$ ) has been measured at wavelength $\lambda_{\mathrm{r}}>\lambda_{0}$. The values for $K_{\mathrm{f}}\left(N_{\mathrm{ss}}, S\right)$ and $K_{\mathrm{r}}\left(\tau_{\mathrm{r}}\right)$ can then be determined from

$$
K_{\mathrm{f}}\left(N_{\mathrm{ss}}, S\right)=\frac{\delta\left(\lambda_{\mathrm{f}}, N_{\mathrm{ss},} S, \tau_{\mathrm{r}}\right)}{\delta_{\mathrm{f}}\left(\lambda_{\mathrm{f}}\right)}
$$

and

$$
K_{\mathrm{r}}\left(\tau_{\mathrm{r}}\right)=\frac{\delta\left(\lambda_{\mathrm{r}}, N_{\mathrm{ss}}, S, \tau_{\mathrm{r}}\right)-K_{\mathrm{f}}\left(N_{\mathrm{ss}}, S\right) \delta_{\mathrm{r}}\left(\lambda_{\mathrm{r}}\right)}{\delta \mathrm{r}\left(\lambda_{\mathrm{r}}\right)}
$$

and these values can be used in eq (1) to interpolate the quantum deficiency between $\lambda_{\mathrm{f}}$ and $\lambda_{\mathrm{r}}$. In the above equations, $\delta_{\mathrm{f}}(\lambda)$ and $\delta_{\mathrm{r}}(\lambda)$ are functions only of wavelength, $\lambda_{f}$ and $\lambda_{\mathrm{r}}$ are the wavelengths at which the internal quantum deficiency is to be normalized, and $\lambda_{0}$ will generally fall between 600 and $650 \mathrm{~nm}$. The subscript $f$ stands for the front of the photodiode since this is the location of the recombination mechanisms that cause $\delta_{\mathrm{f}}(\lambda)$ to exceed zero. The subscript $r$ stands for the rear of the photodiode since this is the location of the recombination mechanism that cause $\delta_{\mathrm{r}}(\lambda)$ to exceed zero. Therefore, $\delta_{\mathrm{f}}(\lambda)$ and $\delta_{\mathrm{r}}(\lambda)$ will be called the front-region and rear-region internal quantum-deficiency spectra, respectively. The functional dependence of $K_{\mathrm{f}}$ and $K_{\mathrm{r}}$ will be dropped from the notation for the remainder of this paper.

Exactly how accurate eqs (1-3) are depends upon the particular type of photodiode and the range covered by the parameters. Figure 1 illustrates the accuracy of eq (1) for the 1337 type photodiode (modeled as described in Part II of this series of papers) over the 400 to $900 \mathrm{~nm}$ spectral region for an internal quantum-deficiency spectrum that is a practical upper limit for this type of photodiode. Figure 1 compares the internal quantum-deficiency spectra $\delta(\lambda, 0,71264 \mathrm{~cm} / \mathrm{s}, \infty)$ and $\delta(\lambda, 0,0,1 \mathrm{~ms})$ with their sum, and with the internal quantum-deficiency spectrum $\delta(\lambda, 0,71264 \mathrm{~cm} / \mathrm{s}, 1$ $\mathrm{ms})$. The latter spectrum and the sum of the two first spectra differ by less than $2 \times 10^{-5}$ over the entire $400 \mathrm{~nm}$ spectral region, and cannot be distinguished at the scale of the figure. (Any errors with absolute values less than $2 \times 10^{-5}$ are negligible for the purposes of this paper.)

Figure 2 illustrates the accuracy of eqs (1-3) over the 400 to $900 \mathrm{~nm}$ spectral region for the case where $\tau_{\mathrm{r}}=\infty$. The spectra in figure 2 were calculated for the range of parameters listed in table 1. 


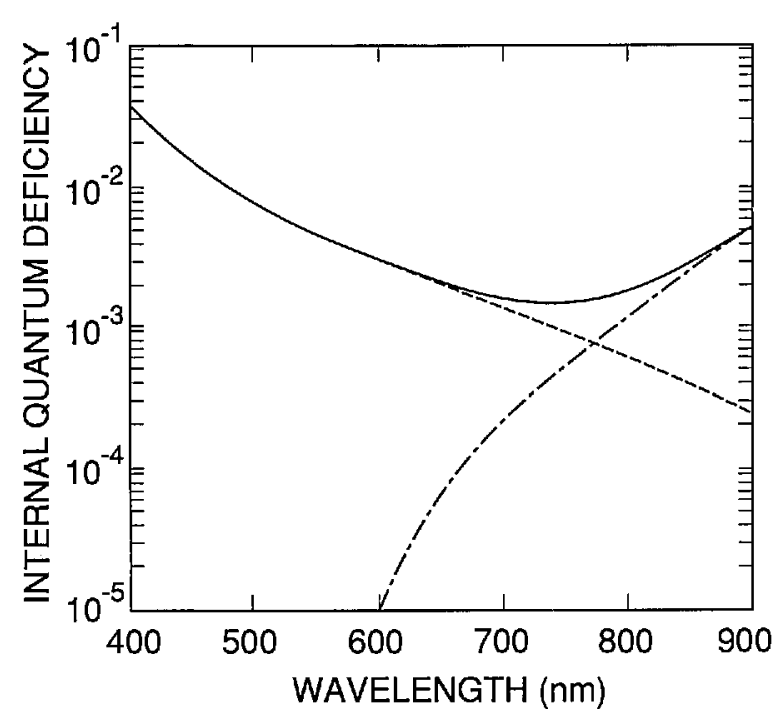

Figure 1. The quantum-deficiency spectra $\delta(\lambda, 0,71264 \mathrm{~cm} / \mathrm{s}$, $\infty)$ (dashed line) and $\delta(\lambda, 0,0,1 \mathrm{~ms}$ ) (dot-dashed line) and thcir sum (solid line).

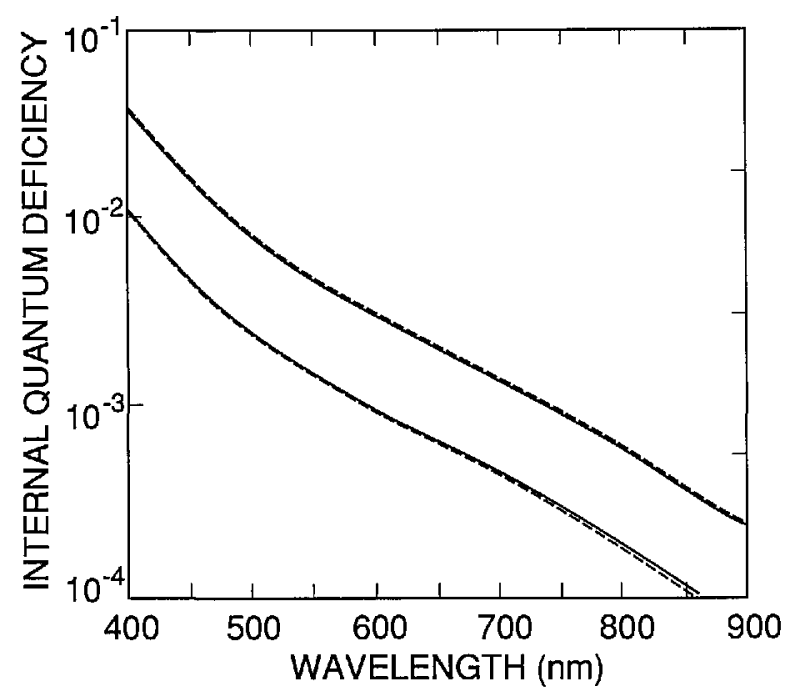

Figure 2. The quantum-deficiency spectra $\delta\left(\lambda, N_{\mathrm{ss}}, S, \tau_{\mathrm{r}}\right)$ for the conditions (Case 1-upper dashed line; Case 2-lower dashed line; Case 3-upper solid line; Case 4-lower solid line) listed in tablc 1.

Table 1. Recombination related parameters used with the doping distributions shown in figure 3 to model the quantumdeficiency spectra of Hamamatsu 1337 type photodiodes

\begin{tabular}{ccccc}
\hline \hline Case & $\begin{array}{c}N_{\text {ss }} \\
\left(\mathrm{cm}^{-3}\right)\end{array}$ & $\begin{array}{c}S \\
\left(10^{5} \mathrm{~cm} / \mathrm{s}\right)\end{array}$ & $\begin{array}{c}\tau_{\mathrm{r}} \\
(\mathrm{s})\end{array}$ & $N_{\mathrm{A}}(x)$ \\
\hline 1 & 0 & 0.71264 & $\infty$ & dashed curve \\
2 & $-3 \times 10^{12}$ & 1.83500 & $\infty$ & dashed curve \\
3 & $-1 \times 10^{13}$ & 100.000 & $\infty$ & dashed curve \\
4 & $-4 \times 10^{12}$ & 1.56000 & $\infty$ & solid curve \\
\hline
\end{tabular}

Cases 1-3 in that table are based on the frontregion doping distribution $N_{\mathrm{A}}(x) / N_{\mathrm{A}}(0)$ shown as the dashed curve in figure 3 , and Case 4 is based on that shown as the solid curve in the same figure. Of six doping distributions measured for different 1337 type photodiodes, the two shown in figure 3 produce the largest change in shape of the internal quantum-deficiency spectrum.

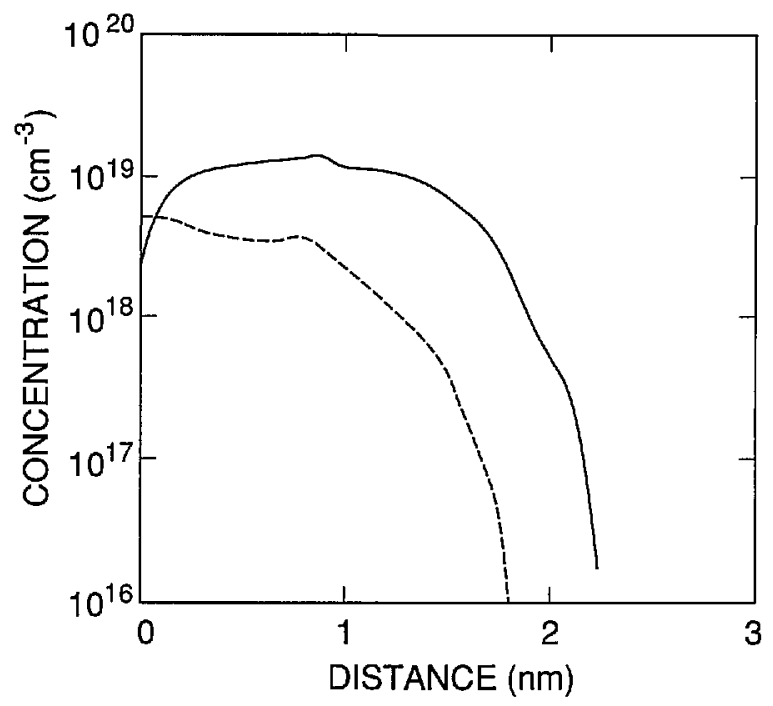

Figure 3. The front-region doping distributions $N_{\mathrm{A}}(x)$ used to calculate the front-region internal quantum-deficiency curves in figure 2.

The shapes of the front-region internal quantum-deficiency spectra in figure 2 are compared in figure 4 by normalizing all of the spectra to 0.01 at $440 \mathrm{~nm}$ and by plotting their differences relative to the normalized spectrum for Case 2. Cases 1 and 3 compared with Case 2 shows how the shape of $\delta_{\mathrm{f}}(\lambda)$ changes when its magnitude is increased by over a factor of three in association with either a substantial decrease or a substantial increase in $N_{\text {ss. }}$. (Cases 1 and 3 bracket a number of cases that were examined in which $N_{s s}$ and $S$ were independently varied within the range of values shown in table 1.)

Case 4 compared with Case 2 shows how the shape of $\delta_{\mathrm{f}}(\lambda)$ changes when the dopant distribution is changed from the dashed curve to the solid curve in figure 4 . This effect is much larger than that associated with varying $N_{\text {ss }}$ and $S$.

The three spectra in figure 4 characterize the uncertainties to be associated with the diode-to-diode variations in $N_{\mathrm{ss}}, S$, and $N_{\mathrm{A}}(x)$ when any one of the spectra in that figure is used as $\delta_{\mathrm{f}}(\lambda)$ with $K_{\mathrm{f}}=0.01$ in eqs (1) and (3). These spectra should be multi- 
plied by $K_{f} / 0.01$ if $K_{f} \neq 0.01$. For the purposes of this paper, the $\delta\left(\lambda, N_{\mathrm{ss}}, S, \infty\right)$ spectrum corresponding to Case 4 in table 1 will be used for $\delta_{\mathrm{f}}(\lambda)$ in eqs (1) and (3).

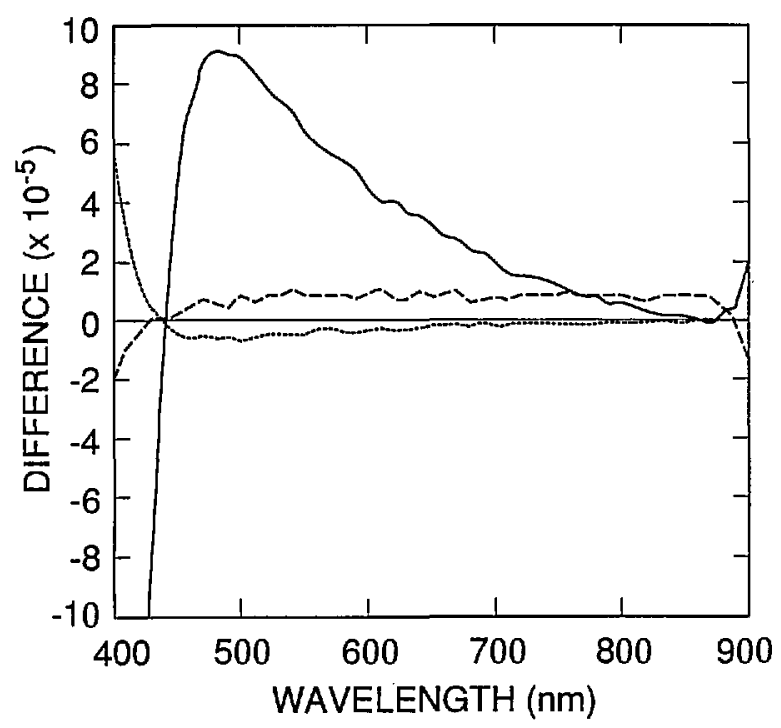

Figure 4. Differences between the internal quantum-deficiency spectra of figure 2 for Cases 1 (dashed line), 3 (dotted line), and 4 (solid line) when normalized to 0.01 at $440 \mathrm{~nm}$, and that for Case 2 when normalized to 0.01 at $440 \mathrm{~nm}$.

The front-region internal quantum deficiency also depends upon two front-region recombination mechanisms not considered above. These are the Auger and band-to-band mechanisms that become important in the heavily doped front region of the photodiode. Both effects were included in all of the spectra described so far by using the default cross sections that are built into PC-1D for these recombination mechanisms. The PC-1D default values for the Auger recombination cross sections give an internal quantum-deficiency spectrum with a value of $9 \times 10^{-6}$ at $400 \mathrm{~nm}$. The shape of this spectrum is well modeled by $\delta_{\mathrm{f}}(\lambda)$ [5]. In fact, an uncertainty of $\pm 100 \%$ can be tolerated in the Auger cross sections without causing an error as large as 2 times $10^{-5}$ anywhere within the 400 to $900 \mathrm{~nm}$ spectral region.

For the doping profile shown as the solid curve in figure 3, the PC-1D default values for the bandto-band recombination cross sections give the lifetimes shown in figure 5 as a function of distance from the oxide-silicon interface in the photodiode. These lifetimes give an internal quantum-deficiency spectrum with a value of $6 \times 10^{-5}$ at $400 \mathrm{~nm}$. This spectrum is not as well modeled by $\delta_{r}(\lambda)$ as is the spectrum for Auger recombination, but an un- certainty of $\pm 50 \%$ in the band-to-band recombination cross sections can be tolerated without raising the error associated with this uncertainty to $2 \times 10^{-5}$ anywhere in the 400 to $900 \mathrm{~nm}$ spectral region.

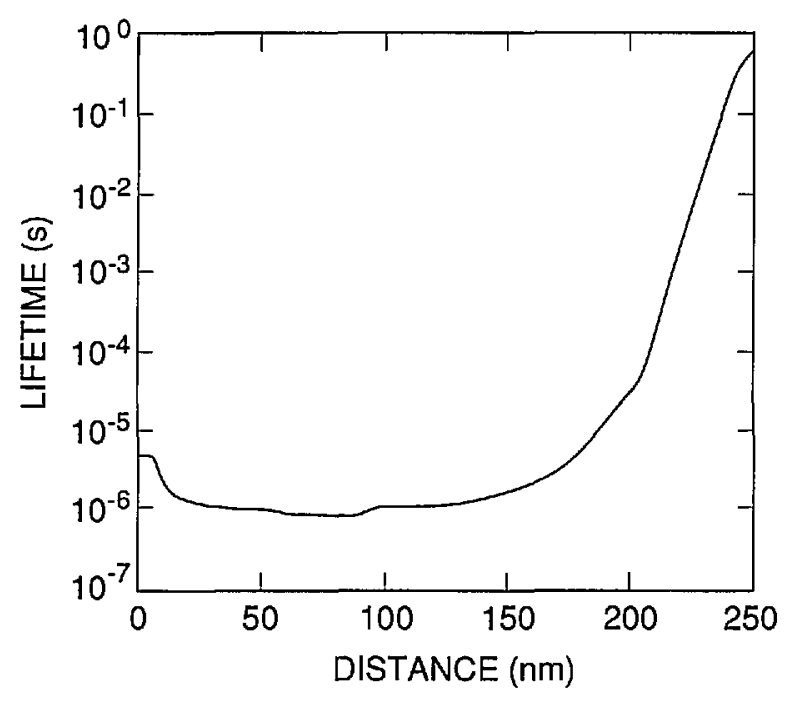

Figure 5. The default, band-to-band recombination lifetime profile calculated by PC-1D for the doping distribution shown as the solid curve in figure 3 .

Now consider $\delta_{\mathrm{r}}(\lambda)$. Figures 6 and 7 illustrate the accuracy of eqs (1) and (3) over the 400 to 900 $\mathrm{nm}$ spectral region for the case where $S=0$. (Note that when $S=0$, the internal quantum-deficiency spectrum is independent of $N_{\text {ss. }}$ ) The spectra in figure 6 were calculated for $\tau_{\mathrm{r}}=1 \mathrm{~ms}$ and $10 \mathrm{~ms}$, with $N_{\mathrm{ss}}=S=0$. For the purposes of this paper, $\delta_{\mathrm{r}}(\lambda)$ will be set to zero in eqs (2) and (3) for $\lambda \leq 610 \mathrm{~nm}$, and the spectrum for $\tau_{\mathrm{r}}=1 \mathrm{~ms}$ will be used for $\delta_{\mathrm{r}}(\lambda)$ for $\lambda>610 \mathrm{~nm}$.

The shapes of the spectra in figure 6 are compared in figure 7 by normalizing them to 0.002 at $860 \mathrm{~nm}$ and plotting their differences relative to the spectrum for $\tau_{\mathrm{r}}=1 \mathrm{~ms}$. The normalization value of 0.002 was chosen as a practical upper limit. The difference spectrum in figure 7 characterizes the uncertainties to be associated with the variations in rear-region lifetime when the spectrum for $\tau_{r}=1$ ms is used as $\delta_{\mathrm{r}}(\lambda)$ with $K_{\mathrm{r}}=0.002$ in eqs (2) and (3). This spectrum should be multiplied by $K_{\mathrm{r}} / 0.002$ if $K_{\mathrm{r}} \not \geq 0.002$.

A convenient function of wavelength has been fitted to the spectrum corresponding to Case 4 in table 1 , and another to the spectrum for $\tau_{\mathrm{r}}=1 \mathrm{~ms}$ in figure 6 . These functions are

$$
\delta_{\mathrm{f}}(\lambda)=A_{0}\left[X(\lambda)+A_{1} X^{2}(\lambda)+A_{2} Y(\lambda)\right],
$$




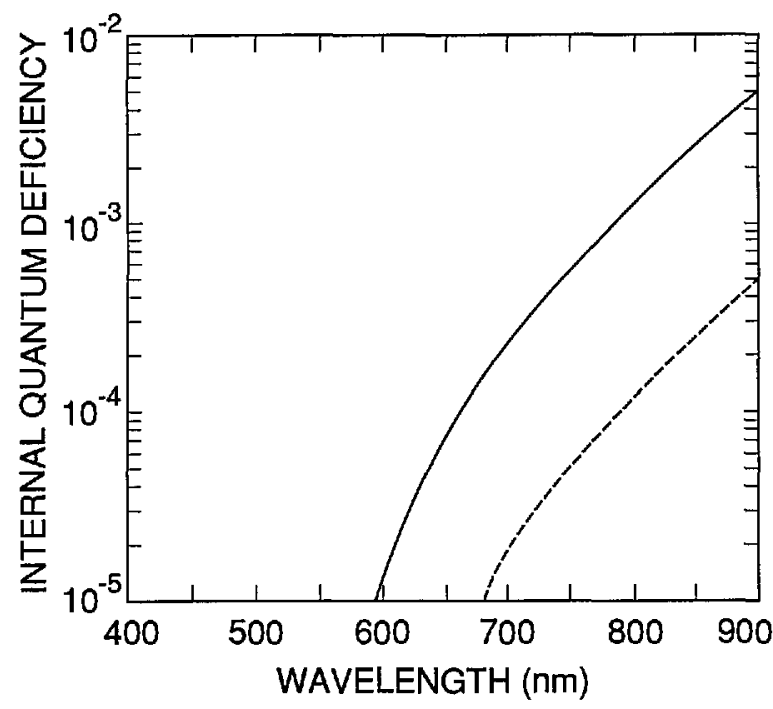

Figure 6. The internal quantum-deficiency spectra $\delta\left(\lambda, N_{\mathrm{ss}}, S, \tau_{\mathrm{r}}\right)$ for $\tau_{\mathrm{r}}=1 \mathrm{~ms}$ (solid line), and $\tau_{\mathrm{r}}=10 \mathrm{~ms}$ (dashed line) with $N_{\mathrm{ss}}=S=0$.

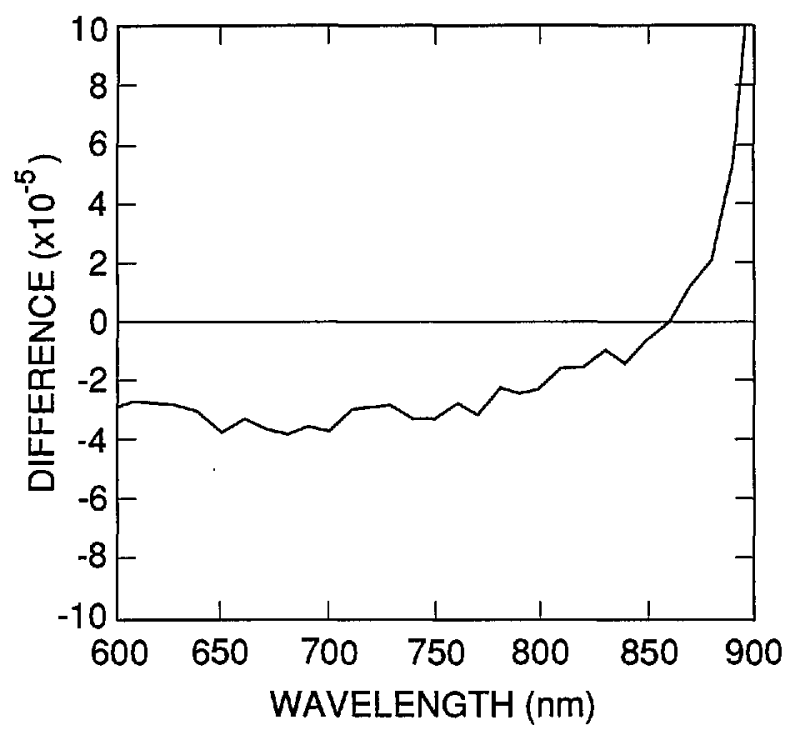

Figure 7. Differences between the internal quantum-deficiency spectra of figure 6 when normalized to 0.002 at $860 \mathrm{~nm}$.

and

$$
\delta_{\mathrm{r}}(\lambda)=A_{0}\left[1 / X(\lambda)-A_{2} / Y(\lambda)\right]
$$

respectively, where

$$
X(\lambda)=\exp \left(-\lambda / \lambda_{0}\right)
$$

and

$$
Y(\lambda)=\exp \left(-\left(\lambda / \lambda_{2}\right)^{2}\right)
$$

The appropriate values for $A_{0}, A_{1}, A_{2}, \lambda_{0}$, and $\lambda_{2}$ for eqs (4) and (5) are listed in table 2 . The function in eq (5) becomes negative for $\lambda<620 \mathrm{~nm}$, in which case eq (5) is replaced with $\delta_{\mathrm{r}}(\lambda)=0$ as mentioned earlier. The values of $A_{0}$ in table 2 normalize $\delta_{\mathrm{f}}(\lambda)$ to 0.01 at $440 \mathrm{~nm}$, and $\delta_{\mathrm{r}}(\lambda)$ to 0.002 at $860 \mathrm{~nm}$, respectively.

With $K_{\mathrm{f}}=0.5516405$, eq (4) fits the spectrum for $N_{\mathrm{ss}}=-3 \times 10^{12} \mathrm{~cm} / \mathrm{s}$ in figure 4 with a residual standard deviation of $2.9 \times 10^{-6}$ over the 420 to $900 \mathrm{~nm}$ spectral region and never differs from that spectrum by more than $7 \times 10^{-6}$ over the same spectral region. This translates to less than $10 \mathrm{ppm}$ when the internal quantum deficiency is normalized to 0.01 at $440 \mathrm{~nm}$; thus the error associated with the use of eq (4) is negligible for the purposes of this paper. The larger variations below $420 \mathrm{~nm}$ are negligible with respect to uncertainties already identified and others discussed below.

With $K_{\mathrm{r}}=1.685081$, eq (5) fits the spectrum shown as a solid line in figure 6 with a residual standard deviation of $1.4 \times 10^{-5}$ over the 610 to 900 $\mathrm{nm}$ spectral region, and never differs from that spectrum by more than $2.5 \times 10^{-5}$ over that same spectral range. This translates to less than $1.7 \times 10^{-5}$ when the quantum deficiency is normalized to 0.002 at $440 \mathrm{~nm}$; thus the error associated with the use of eq (5) is also negligible for the purposes of this paper.

Table 2. Values of the parameters to be used in eq (4) for extrapolating a short wavelength internal quantum-deficiency value to longer wavelengths and in eq (5) for extrapolating a long wavelength internal quantum-deficiency value to shorter wavelengths

\begin{tabular}{clc}
\hline \hline Parameter & \multicolumn{1}{c}{ eq (4) } & eq (5) \\
\hline$A_{0}$ & $1.38002 \times 10^{2}$ & $2.02157 \times 10^{-8}$ \\
$A_{1}$ & $1.47530 \times 10^{2}$ & $3.51764 \times 10^{3}$ \\
$A_{2}$ & $8.07476 \times 10^{-5}$ & 0.0 \\
$\lambda_{4}$ & $4.39312 \times 10^{1} \mathrm{~nm}$ & $7.53991 \times 10^{1} \mathrm{~nm}$ \\
$\lambda_{2}$ & $4.27998 \times 10^{2} \mathrm{~nm}$ & $1.66889 \times 10^{8} \mathrm{~nm}$ \\
\hline
\end{tabular}

\section{Errors Associated with Quantum- Deficiency Calculations}

Two types of error associated with the quantumdeficiency values calculated by PC-1D can be distinguished: 1) numerical errors associated with the algorithms used by $\mathrm{PC}-1 \mathrm{D}$ and the limited number of finite elements available to PC-1D, and 2) physical errors associated with approximations 
and simplifications in the physical models used with, or built into, PC-1D. Both types are considered in this section.

It was shown in reference [5] that $\delta_{\mathrm{f}}(\lambda)$ is well approximated by

$$
\begin{aligned}
& \delta_{\mathrm{f}}(\lambda)=\frac{\Gamma\{I(0)-I[\alpha(\lambda)]\}}{1+\Gamma[\alpha(\lambda)]} \\
& I[\alpha(\lambda)] \int_{0}^{x_{1}} \hat{k}(x) \exp (-\alpha(\lambda) x) \mathrm{d} x, \\
& \hat{k}(x)=k(x) / k(0) \\
& k(x)=1 /\left[m_{0}(x) D_{m}(x)\right] \\
& \Gamma=S / D_{m}(0)
\end{aligned}
$$

where $\alpha(\lambda)$ is the absorption-coefficient spectrum of silicon, $x$ is the distance from the oxide-silicon interface toward the rear of the photodiode, $m_{0}(x)$ is the equilibrium minority-carrier concentration at the point $x$ in the front region of the photodiode, and $D_{m}(x)$ is the equilibrium diffusion constant for the minority carriers at the point $x$. Note that the sign in the numerator in eq (8) is correct and that the sign is incorrect in reference [5]. Also, note that $f(\lambda)$ becomes very insensitive to $x_{1}$ as $x_{1}$ increases beyond some critical value. The point where the electron and hole concentrations cross is a convenient choice for $x_{1}$, but smaller values would work as well.

From the point of view of PC-1D, the kernel $k(x)$ in eq (11) is given by

$$
k(x)=q M_{0}(x) /\left[n_{i e}^{2}(x) k T \mu_{\mathrm{m}}(x)\right],
$$

where $M_{0}(x)$ is the equilibrium majority carrier concentration in the front region, $n_{\mathrm{ie}}(x)$ is the effective intrinsic carrier concentration [6], which in PC-1D includes the effect of Fermi-Dirac statistics, $\mu_{\mathrm{m}}(x)$ is the minority-carrier mobility as a function of position in the front region, $k$ is the Boltzmann constant, and $T$ is the temperature of the photodiode.

Figure 8 compares $\exp (-\alpha(400 \mathrm{~nm}) x)$ with the equilibrium functions $M_{0}(x), n_{\mathrm{ie}}(x)$, and $\mu_{\mathrm{m}}(x)$ as calculated by PC-1D over the range $0<x<200 \mathrm{~nm}$ for the same photodiode model used to calculate the quantum-deficiency spectra in figure 3 for Case 2 of table 1. Equations (8-12), figure 8, and figure 3 show why the shape of $\delta_{\mathrm{f}}(\lambda)$ is very insensitive to $N_{\text {ss }}$ while being quite sensitive to the shape of $N_{\mathrm{A}}(x) / N_{\mathrm{A}}(0)$. Since the width of the accumulation layer created by $N_{\mathrm{ss}}$ is small compared to the dis- tance over which $\exp (-\alpha(\lambda) x)$ changes significantly for $\lambda \geq 400 \mathrm{~nm}$, changes in $N_{\mathrm{ss}}$ have the effect of multiplying the integral in eq (9) by a constant factor independent of $\lambda \geq 400 \mathrm{~nm}$. On the other hand, the changes in $N_{\mathrm{A}}(x) / N_{\mathrm{A}}(0)$ shown in figure 3 extend well into the region where $\exp (-\alpha(\lambda) x)$ varies significantly from unity for $\lambda=400 \mathrm{~nm}$. As $\lambda$ is increased above $400 \mathrm{~nm}$, the shape of $\delta_{\mathrm{f}}(\lambda)$ will become less dependent on the shape of $N_{A}(x)$ / $N_{\mathrm{A}}(0)$ because $\alpha(\lambda)$ decreases with increasing $\lambda$.

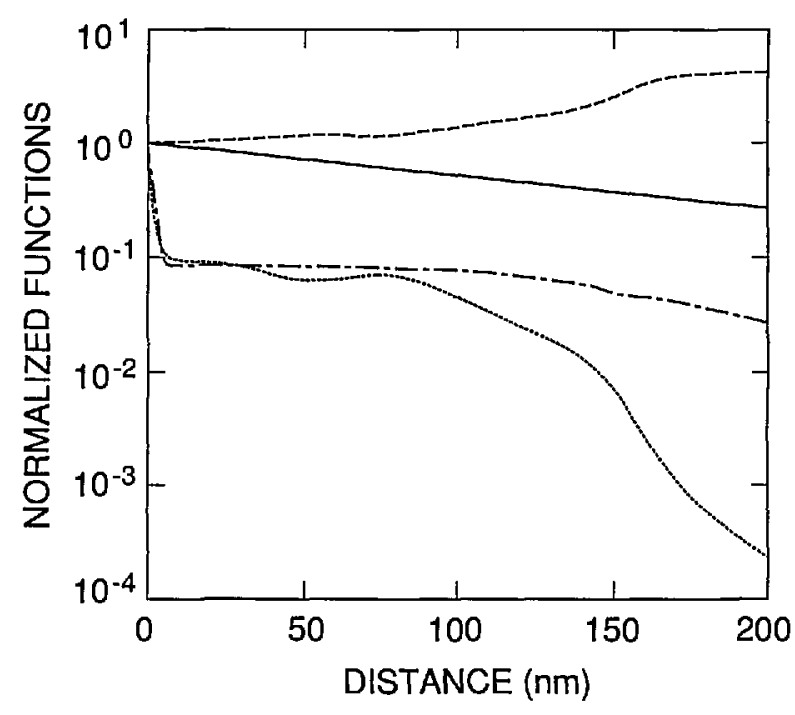

Figure 8. Comparison of $\hat{M}_{0}(x)$ (dotted line), $\hat{n}_{\mathrm{je}}(x)$ (dashed line), $\hat{\mu}$ (dot-dashed line), and $\exp (-\alpha(400 \mathrm{~nm}) x$ ) (solid line) for a 1337 type photodiode with $N_{\mathrm{ss}}, S$, and $\tau_{\mathrm{r}}$ set as defined in Case 2 of table 1. The tildes in the preceding expressions indicate that these expressions have been normalized to unity at $x=0$, as shown for $k(x)$ in eq (10).

The numerical accuracy with which PC-1D calculates $M_{0}(x)$ within the framework of the driftdiffusion approximations for the conditions of charge accumulation at an oxide-silicon interface as shown in figure 8 has been studied previously and found to be quite good [7]. With a uniform doping density of $10^{19} \mathrm{~cm}^{-3}$ and an oxide charge density of $10^{13} \mathrm{~cm}^{-2}$, PC-1D overestimates $M_{0}(x)$ by about $9 \%$ at $x=0$, decreasing to within $\pm 1 \%$ for $x>1 \mathrm{~nm}$. The uncertainty in $k(x)$ due to this source of error will be modeled by replacing $k(x)$ by

$$
k_{1}(x)=k(x) /\left(1-0.1 x / x_{0}\right)
$$

for $x<x_{0}$, and

$$
k_{1}(x)=k(x) / 0.9
$$


for $x>x_{0}$ in the kernel of the integral in eq (9), where $x_{0}=1 \mathrm{~nm}$.

Another source of error that must be considered is the numerical accuracy with which PC-1D would calculate the steady-state photocurrent if the equilibrium carrier concentration were exact. The uncertainty associated with this source of error was estimated as follows: 1) The internal quantumdeficiency spectrum was calculated directly from the PC-1D solution of the steady-state driftdiffusion equations for the total current flowing in the photodiode for the conditions described by Case 2 of table 1. 2) Equations (8-13) were used to calculate the internal quantum-deficiency spectrum from the equilibrium values of $M_{0}(x)$, $n_{\text {ic }}(x)$, and $\mu_{\mathrm{m}}(x)$ calculated by PC-1D for the same conditions as in 1) above. The integration in eq (9) was carried out using a generalization of Simpson's rule for nonequidistant points. 3) Both spectra were normalized to 0.01 at $440 \mathrm{~nm}$, and the differences calculated. There is no reason to believe that these differences, which exceed $2 \times 10^{-5}$ only at 400 and at $900 \mathrm{~nm}$, are caused by errors associated with PC-1D. It is just as likely that they are associated with eqs (8-12) since these equations were derived from a number of assumptions that are not rigorously satisfied. The important point is that it is unlikely that either calculation is in error by much more than the differences between them. This verifies the numerical accuracy of the photocurrents calculated by PC-1D to the level of accuracy required in this paper.

There are a number of other errors, which are associated with incomplete or approximate physical models built into PC-1D, that also affect the accuracy with which $P C-1 D$ calculates the functions defining $k(x)$ in eq (13). These include quantummechanical effects (tunneling) [8] that change the shape of $M_{0}(x)$ from that calculated from the driftdiffusion approximation for $x<1 \mathrm{~nm}$, band-gap narrowing effects [9] associated with heavy doping and large surface-fields [10] that cause $n_{\text {ie }}(x)$ [6] to depend upon position in the photodiode, and carrier-carrier and carrier-ion scattering mechanisms that cause $\mu_{\mathrm{m}}(x)$ to depend upon position [11]. It is beyond the scope of this paper to investigate each of these sources of error in detail, but the uncertainty in $k(x)$ due to this source of error will be modeled by replacing $k(x)$ by

$$
k_{2}(x)=k(x)\{1-0.1 \log [\hat{k}(x)]\}
$$

in the kernel of the integral in eq (9). This allows an error of $10 \%$ per decade change in $k(x)$.
The differences between the internal quantumefficiency spectrum calculated from eqs $(8-13)$ by replacing $k(x)$ with either $k_{1}(x)$ or $k_{2}(x)$ in eq (9) and the spectrum calculated using $k(x)$ were calculated after all three spectra were normalized to 0.01 at $440 \mathrm{~nm}$. Neither difference exceeded $2 \times 10^{-5}$ over the entire 400 to $900 \mathrm{~nm}$ spectral region. Therefore, all of these sources of error are considered negligible for the purposes of this paper.

Another source of error that is obvious in eqs $(8-12)$ is the uncertainty associated with the absorption-coefficient data used in the simulations. As described in Part $I$, the absorption-coefficient data were calculated from an equation [12] fitted to the data of Weakliem and Redfield [13]. An alternate set of absorption-coefficient data was described by Philipp [14]. Figure 9 plots the differences between the internal quantum-deficiency spectra normalized to 0.01 at $440 \mathrm{~nm}$ for Case 2 of table 1 when based on the absorption-coefficient data in reference [14] and when based on the equation in reference [12]. These differences will be used as the estimated uncertainties arising from the uncertainties in the silicon absorption-coefficient spectrum when $\delta_{\mathrm{f}}(\lambda)$ is used to extrapolate an internal quantum deficiency of 0.01 from $440 \mathrm{~nm}$ to longer wavelengths with a 1337 type photodiode. A similar calculation was carried out for the case where $S=0$ and $\tau_{\mathrm{r}}=1 \mathrm{~ms}$. Both spectra were normalized to 0.002 at $860 \mathrm{~nm}$. The differences, which are shown in figure 10 , are taken as the estimated uncertainties arising from the uncertainties in the silicon absorption-coefficient spectrum where $\delta_{\mathrm{r}}(\lambda)$ is used to extrapolate an internal quantum deficiency of 0.002 from $860 \mathrm{~nm}$ to shorter wavelengths with a 1337 type photodiode.

Figures 11 and 12 plot the quadrature sum of the difference spectra plotted in figures 4 and 9 and in figures 7 and 10, respectively. The spectrum in figure 11 plots the estimated (one standard deviation) uncertainty as a function of wavelength that is associated with the use of $\delta_{\mathrm{f}}(\lambda)$ in eq (4) to extrapolate a measured internal quantum deficiency of 0.01 from $440 \mathrm{~nm}$ to longer wavelengths. The major sources of error contributing to the uncertainty spectrum in figure 11 are the diode-to-diode variations in $N_{\mathrm{A}}(x) / N_{\mathrm{A}}(0)$ and the uncertainty in the absorption-coefficient spectrum for silicon, but a number of other sources of error were identified with the help of eqs (8-12), and any that produced differences greater than $2 \times 10^{-5}$ for $400 \mathrm{~nm}$ $<\lambda<900 \mathrm{~nm}$ are included in figure 11 . 


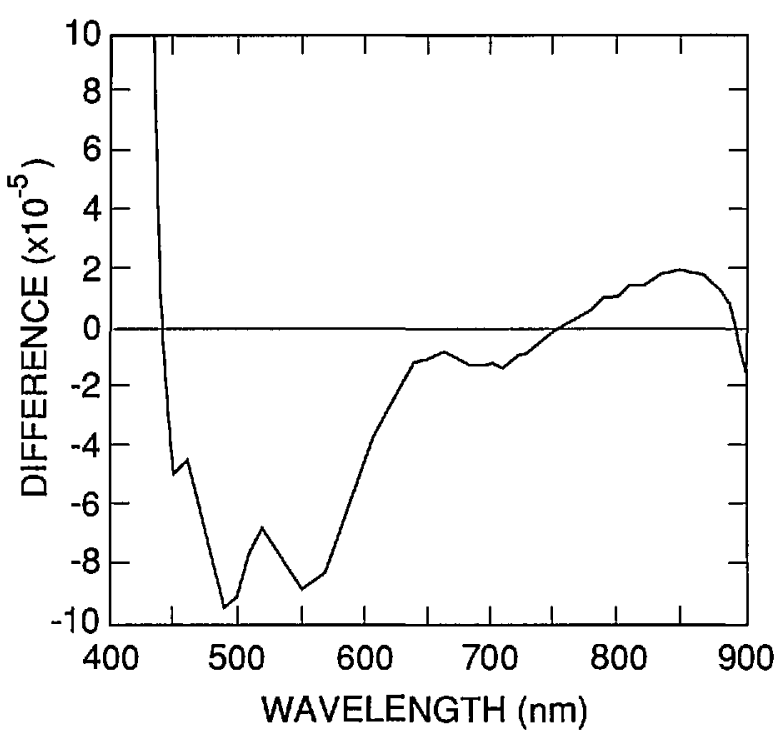

Figure 9. Differenees between the internal quantum-deficiency spectrum for a 1337 type photodiode satisfying the conditions defined in table 1 for the absorption-coefficient data of reference [14] and for the absorption-coefficient data of reference [12].

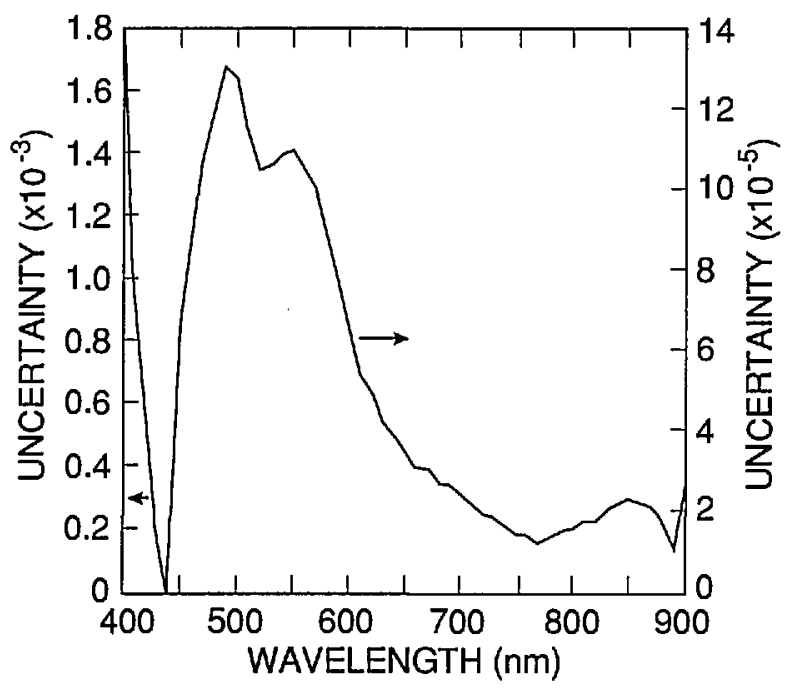

Figure 10. Differences between the internal quantum-deficiency spectra for a 1337 type photodiode with $S=0$ and $\tau_{\mathrm{r}}=1 \mathrm{~ms}$ for the absorption-coefficient data of reference [14] and for the absorption-coefficient data of reference [12].

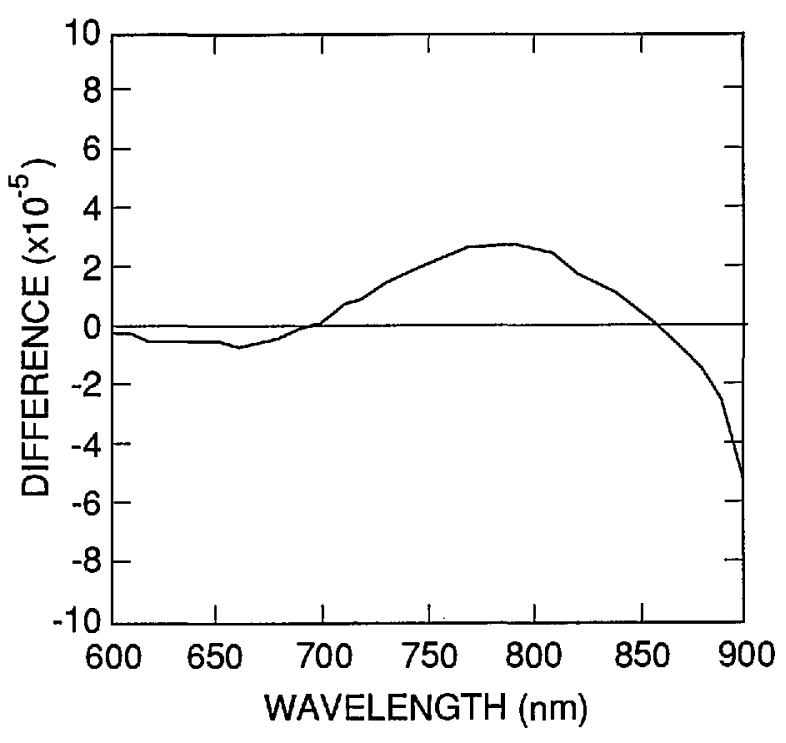

Figure 11. Sum in quadrature of the curves in figures 4 and 9. This curve is the nominal uncertainty associated with the use of eq (4) in eq (2) to extrapolate a quantum deficiency of 0.01 at 440 $\mathrm{nm}$ to any other wavelength between 400 and $900 \mathrm{~nm}$. Notice that the sum is plotted on different scales below and above 440 nm.

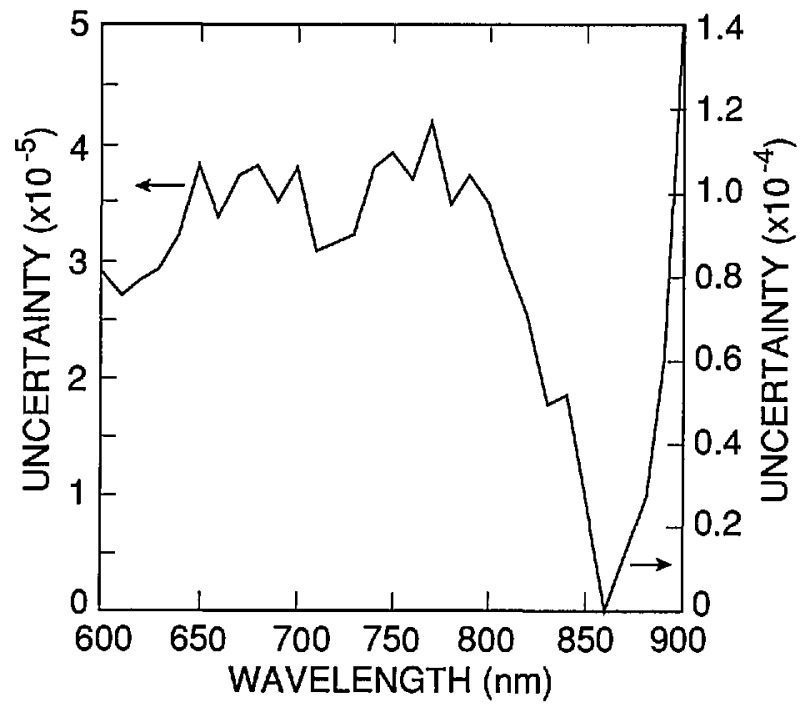

Figure 12. Sum in quadrature of the curves in figures 7 and 10. This curve is the nominal uncertainty associated with the use of eq (5) in eq (2) to extrapolate a quantum deficiency of 0.02 at 860 $\mathrm{nm}$ to any other wavelength between 400 and $900 \mathrm{~nm}$. Notice that the sum is plotted on different scales below and above 440 nm. 
The spectrum in figure 12 plots the estimated (one standard deviation) uncertainty as a function of wavelength that is associated with the use of $\delta_{\mathrm{r}}(\lambda)$ in eq (5) to extrapolate a measured internal quantum deficiency of 0.002 from $860 \mathrm{~nm}$ to shorter wavelengths. The major sources of error contributing to the uncertainty spectrum in figure 12 are the change in shape of $\delta_{\mathrm{r}}(\lambda)$ with $\tau_{\mathrm{r}}$ and the uncertainty in the absorption-coefficient spectrum for silicon. All other sources of error considered were negligible with respect to $2 \times 10^{-5}$ for $400 \mathrm{~nm}$ $<\lambda<900 \mathrm{~nm}$.

No equivalents to eqs (8-12) were used to guide the error analysis for $\delta_{\mathrm{r}}(\lambda)$. The results of the uncertainty analysis of $\delta_{\mathrm{f}}(\lambda)$ show that numerical accuracy of PC-1D will be a negligible source of error. This leaves the errors associated with the approximations in the physical models that are built into PC-1D. Some of these were tested, but no tests were possible for the use of a single SRH trap level to model the recombination in the rear region of the photodiode. This approximation could be a non-negligible source of error; it was shown in Part I that this approximation introduced errors of the order of the effect being modeled in nonlinearity simulations on a UV444B type photodiode. A more complete model might make $\tau_{\mathrm{r}}$ depend upon position in the photodiode, which might modify the shape of $\delta_{\mathrm{r}}(\lambda)$ and add an uncertainty that is not included in figure 12 .

\section{Comparison with Experiment}

To use the results developed thus far, it is necessary to define the internal quantum-deficiency interpolating function $\delta(\lambda)$, and to assume that it exactly satisfies eqs (1-3), which can be rewritten in more compact form as

$$
\begin{aligned}
& \delta(\lambda)= \\
& \delta_{x}\left(\lambda_{f}\right) \frac{\delta_{f}(\lambda)}{\delta_{f}\left(\lambda_{f}\right)}+\delta_{x}\left(\lambda_{r}\right)\left[1-\frac{\delta_{f}\left(\lambda_{r}\right)}{\delta_{r}\left(\lambda_{r}\right)}\right] \frac{\delta_{r}(\lambda)}{\delta_{r}\left(\lambda_{r}\right)},
\end{aligned}
$$

where $\delta_{\mathrm{x}}\left(\lambda_{f}\right)$ and $\delta_{\mathrm{x}}\left(\lambda_{\mathrm{r}}\right)$ are the measured values of the internal quantum deficiency at the wavelengths $\lambda_{\mathrm{f}}$ and $\lambda_{\mathrm{r}}$, respectively; $\delta_{\mathrm{f}}(\lambda)$ and $\delta_{\mathrm{r}}(\lambda)$ are defined in eqs (4) and (5), respectively, and the constants used in these equations are defined in table 2 . The uncertainty associated with $\delta(\lambda)$ due to the sources of error considered in the last two sections of this paper can be obtained by summing in quadrature each of the terms on the right-hand side of the differential of eq (17). That differential is given by

$$
\begin{aligned}
& d \delta(\lambda)=d \delta_{\mathrm{x}}\left(\lambda_{\mathrm{f}}\right)\left[\frac{\delta_{\mathrm{f}}(\lambda)}{\delta_{\mathrm{f}}\left(\lambda_{\mathrm{f}}\right)}\right] \\
& +d \delta_{\mathrm{x}}\left(\lambda_{\mathrm{r}}\right)\left[\frac{\delta_{\mathrm{r}}(\lambda)}{\delta_{\mathrm{r}}\left(\lambda_{\mathrm{r}}\right)}\right]\left[1-\frac{\delta_{\mathrm{f}}\left(\lambda_{\mathrm{r}}\right)}{\delta_{\mathrm{r}}\left(\lambda_{\mathrm{r}}\right)}\right] \\
& +d \delta_{\mathrm{f}}(\lambda)\left[\frac{\delta_{\mathrm{x}}\left(\lambda_{\mathrm{f}}\right)}{\delta_{\mathrm{f}}\left(\lambda_{\mathrm{f}}\right)}\right] \\
& +d \delta_{\mathrm{r}}(\lambda)\left[\frac{\delta_{\mathrm{x}}\left(\lambda_{\mathrm{r}}\right)}{\delta_{\mathrm{r}}\left(\lambda_{\mathrm{r}}\right)}\right]\left[1-\frac{\delta_{\mathrm{f}}\left(\lambda_{\mathrm{r}}\right)}{\delta_{\mathrm{r}}\left(\lambda_{\mathrm{r}}\right)}\right] .
\end{aligned}
$$

The differentials $d \delta_{\mathrm{f}}(\lambda)$ and $d \delta_{\mathrm{r}}(\lambda)$ are plotted in figures 11 and 12 for $\lambda_{\mathrm{f}}=440 \mathrm{~nm}$, and for $\lambda_{\mathrm{f}}=860$ $\mathrm{nm}$, respectively.

There are not many data available against which to test eqs (17) and (18), but there are some. For instance, Zalewski and Hoyt [4] have reported the internal quantum efficiencies at $441.6 \mathrm{~nm}$ and the spectral responsivities and absorptances at 633.0 $\mathrm{nm}$ for five multiple reflection (trap) [4,15-17] radiometers based on Hamamatsu 1337 photodiodes at 441.6 and $633.0 \mathrm{~nm}$. Similarly, Fox [17] has reported the average internal quantum efficiency of 10 trap radiometers also based on Hamamatsu 1337 photodiodes at six $\mathrm{Ar}^{+}$ion laser lines, and he has also measured the oxide-bias correction for a single Hamamatsu 1337 photodiode at four $\mathrm{Ar}^{+}$ ion laser lines. Zalewski and Hoyt [4] presented their data, which are summarized in bold face type in table 3, as an intercomparison of spectral responsivity. Since the internal quantum deficiency is the quantity of interest in this paper, it is necessary to compute this quantity from the data reported by Zalewski and Hoyt as shown in table 3. The corrections for nonlinearity that Zalewski and Hoyt derived from reverse-bias measurements also eliminate any effects of recombination in the rear region of the photodiodes.

Table 3. Average values of, and uncertainty estimates for, the internal quantum efficiency $\epsilon_{\mathrm{f}}$, spectral responsivity $R$, one minus the refleetance $(1-\rho)$ and nonlinearity correction $(1-N L)$ reported in reference [4] (bold face type) at wavelength $\lambda$ for five multiple-reflection radiometers based on 1337 type photodiodes, and internal quantum-deficiency values $\delta_{x}(\lambda)$ calculated from the reported data

\begin{tabular}{cccccc}
\hline \hline$\lambda$ & $\epsilon_{\mathrm{f}}$ & $\begin{array}{c}R \\
(\mathrm{~A} / \mathrm{W})\end{array}$ & $(1-\rho)$ & $(1-\mathrm{NL})$ & $\delta_{x}(\lambda)$ \\
$(\mathrm{nm})$ & & & & \\
\hline 441.6 & +0.99347 & & & & +0.00653 \\
& \pm 0.00040 & & & \multicolumn{2}{c}{ \pm 0.00040} \\
633.0 & +0.50840 & +0.99704 & $\mathbf{+ 0 . 9 9 9 7 2}$ & +0.00097 & \\
& & \pm 0.00009 & \pm 0.00014 & \pm 0.00004 & \pm 0.00023
\end{tabular}


Fox's [17] oxide-bias data, which are summarized in table 4, were measured at $10 \mathrm{~V}$, and were reported as a fractional increase in photocurrent $I$ given by $\gamma_{0}=I(10 \mathrm{~V}) / I(0)-1$. The oxide-bias experiment reported in Part II shows that the internal quantum efficiency at zero bias $\delta_{\mathrm{x}}$ is given by

$$
\delta_{x}=1-1 /\left(1+1.13 \gamma_{0}\right)
$$

where the maximum oxide bias-voltage is $10 \mathrm{~V}$. The average internal quantum efficiencies reported by Fox are also shown in table 4, and the internal quantum deficiencies calculated from them are also shown there.

Table 4. Oxide-bias data $\gamma_{0}$ reported in referenee [17] for a single 1337 type photodiode, the average internal quantum-effieiency data $\epsilon_{x}$ reported in referenee [17] for ten multiple-refleetion radiometers based on 1337 type photodiodes, and the quantum defieieneies $\delta_{x}(\lambda)$ caleulated from these sets of data

\begin{tabular}{ccccc}
\hline \hline$\lambda$ & \multicolumn{2}{c}{ oxide bias } & \multicolumn{2}{c}{ iqe } \\
$(\mathrm{nm})$ & $\gamma_{0}$ & $\delta_{x}(\lambda)$ & $\epsilon_{x}$ & $\delta_{x}(\lambda)$ \\
\hline 406.85 & +0.0126 & +0.01404 & +0.9867 & +0.0133 \\
& \pm 0.00015 & \pm 0.00017 & \pm 0.0002 & \pm 0.0002 \\
468.18 & +0.0044 & +0.00495 & +0.9934 & +0.0066 \\
& \pm 0.00015 & \pm 0.00017 & \pm 0.0003 & \pm 0.0003 \\
568.35 & & & +0.9977 & +0.0023 \\
& & & \pm 0.00020 & \pm 0.0002 \\
647.30 & +0.0007 & +0.00079 & +0.9986 & +0.0014 \\
& \pm 0.00015 & \pm 0.00017 & \pm 0.0002 & \pm 0.0002 \\
799.54 & & & +0.9989 & +0.0011 \\
& & & \pm 0.0002 & \pm 0.0002 \\
859.07 & +0.0000 & +0.00000 & +0.9987 & +0.0013 \\
& \pm 0.00015 & \pm 0.00017 & \pm 0.0002 & \pm 0.0002 \\
& & & & \\
\hline
\end{tabular}

Equation (17) was normalized to the results of Zalewski and Hoyt at $\lambda_{\mathrm{f}}=441.6 \mathrm{~nm}$, and the predictions of that equation are compared with the results of Zalewski and Hoyt at $633 \mathrm{~nm}$ in table 5 . Note that $\delta_{x}\left(\lambda_{r}\right)=0$ for this data set due to the application of reverse bias. Equation (17) was also normalized to the oxide-bias results of Fox at $\lambda_{\mathrm{f}}=468.18 \mathrm{~nm}$, and the predictions of that equation are compared with the oxide-bias results in table 6 . Again note that $\delta_{x}\left(\lambda_{r}\right)=0$ because oxide-bias measurements are not sensitive to recombination in the rear of the photodiode.

The uncertainty estimates for eq (17) in tables 5 and 6 made use of the data in figure 11 even though those data apply to $\lambda_{f}=440 \mathrm{~nm}$ rather than 441.6 or $468.2 \mathrm{~nm}$. This does not distort the esti- mated uncertainty significantly because the terms are added in quadrature, and the first term on the right-hand side of eq (18) is larger than the second term for both sets of data. Fox's data point at $406.85 \mathrm{~nm}$ was included in table 6 even though the uncertainties associated with extrapolating $\delta_{\mathrm{f}}(\lambda)$ to wavelengths shorter than $\lambda_{\mathrm{f}}$ are very unfavorable for high-accuracy applications. The good agreement at this wavelength must be considered fortuitous considering the uncertainty associated with the predicted value. The differences at the other wavelengths never exceed 0.00015 , and they fall within the estimated uncertainty for the differences.

Table 5. Comparison of predietions of eq (17) with measured internal quantum-defieiency data $\delta_{x}(\lambda)$ in table 3 for $\lambda_{f}=441.6$ $\mathrm{nm}$ and $\delta_{x}\left(\lambda_{\mathrm{r}}\right)=0$

\begin{tabular}{llll}
\hline $\begin{array}{l}\text { Predietion } \\
\text { wavelength } \\
(\mathrm{nm})\end{array}$ & $\begin{array}{c}\text { Measured } \\
\text { value } \\
\text { referenee [4] }\end{array}$ & $\begin{array}{c}\text { Predicted } \\
\text { value } \\
\text { eq (17) }\end{array}$ & Difference \\
\hline 633.0 & +0.00097 & +0.00089 & -0.00008 \\
& \pm 0.00023 & \pm 0.00007 & \pm 0.00024 \\
\hline
\end{tabular}

Table 6. Comparison of predietions of eq (17) with intemal quantum-defieiency data $\delta_{x}(\lambda)$ derived from oxide-bias data in table 4 for $\lambda_{f}=468.18 \mathrm{~nm}$ and $\delta_{x}\left(\lambda_{r}\right)=0$

\begin{tabular}{llll}
\hline $\begin{array}{c}\text { Predietion } \\
\text { wavelength } \\
(\mathrm{nm})\end{array}$ & $\begin{array}{c}\text { Measured } \\
\text { value } \\
\text { referenee [15] }\end{array}$ & $\begin{array}{c}\text { Predieted } \\
\text { value } \\
\text { eq (17) }\end{array}$ & Differenee \\
\hline 406.85 & +0.01404 & +0.01321 & -0.00083 \\
& \pm 0.00017 & \pm 0.01144 & \pm 0.01144 \\
647.30 & +0.00079 & +0.00090 & +0.00010 \\
& \pm 0.00017 & \pm 0.00030 & \pm 0.00034 \\
859.07 & +0.00000 & +0.00015 & +0.00015 \\
& \pm 0.00017 & \pm 0.00005 & \pm 0.00018 \\
\hline
\end{tabular}

Table 7 and figure 13 compare the internal quantum-deficiency results of Fox [17] with the predictions of eq (17) when normalized at $\lambda_{\mathrm{f}}=468.18 \mathrm{~nm}$ and at $\lambda_{\mathrm{r}}=859.07 \mathrm{~nm}$. The data in figures 11 and 12 that apply for $\lambda_{\mathrm{f}}=440 \mathrm{~nm}$ and for $\lambda_{\mathrm{r}}=860 \mathrm{~nm}$, respectively, are used with eq (18) to estimate the uncertainties assigned to the predictions of eq (17). The difference at $406.85 \mathrm{~nm}$ is much larger than that obtained with the oxide-bias data but still falls within the estimated uncertainty. Because the oxide-bias experiment is not sensitive to the quantum yield for electron-hole pair production, it is tempting to imagine that the discrepancy 
Table 7. Comparison of predictions of eq (17) with measured internal quantum-deficiency data in table 4 for $\lambda_{\mathrm{f}}=468.18 \mathrm{~nm}$ and $\lambda_{\mathrm{r}}=859.07 \mathrm{~nm}$

\begin{tabular}{llll}
\hline \hline $\begin{array}{l}\text { Prcdiction } \\
\text { wavclength } \\
(\mathrm{nm})\end{array}$ & $\begin{array}{c}\text { Measured } \\
\text { value } \\
\text { reference }[15]\end{array}$ & $\begin{array}{c}\text { Predicted } \\
\text { value } \\
\text { eq }(17)\end{array}$ & Difference \\
\hline $406.85 \mathrm{~nm}$ & +0.0133 & +0.01744 & +0.00414 \\
& \pm 0.00025 & \pm 0.01000 & \pm 0.01000 \\
$568.35 \mathrm{~nm}$ & +0.0023 & +0.00224 & -0.00006 \\
& \pm 0.00030 & \pm 0.00007 & \pm 0.00031 \\
$647.30 \mathrm{~nm}$ & +0.0014 & +0.00121 & -0.00019 \\
& \pm 0.00020 & \pm 0.00004 & \pm 0.00020 \\
$799.54 \mathrm{~nm}$ & +0.0011 & +0.00082 & -0.00028 \\
& \pm 0.00021 & \pm 0.00009 & \pm 0.00023 \\
\hline
\end{tabular}

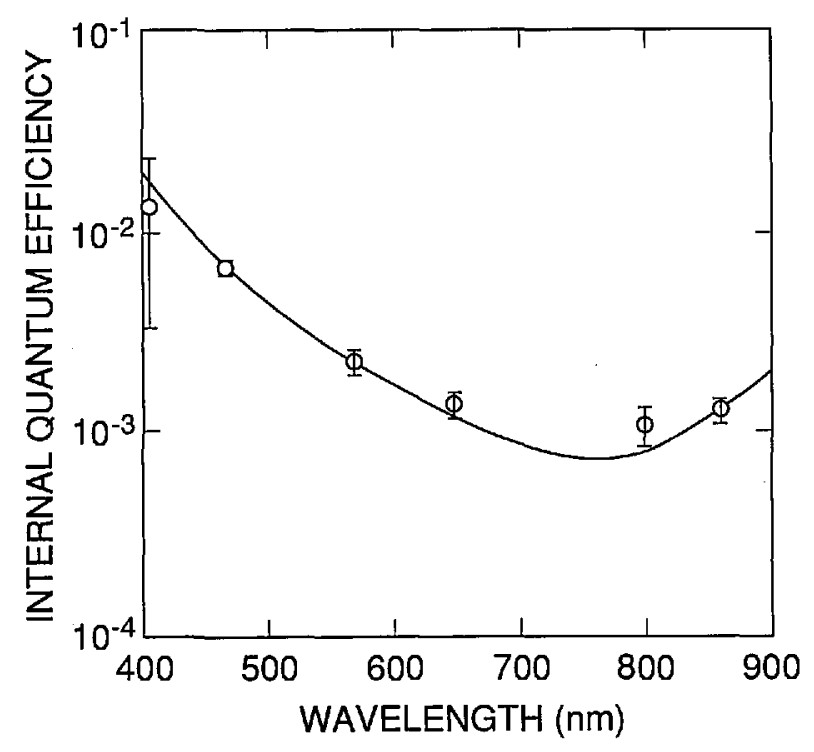

Figure 13. Comparison of measured data of reference [17] (open circles) with eq (17) (solid linc) normalized to pass through the measured point at 468.18 and $859.1 \mathrm{~nm}$. At these wavelengths the error bars are the uncertainties in the measured values; at the other wavelengths the error bars are the quadrature sum of the uncertainties in the measured values and those associated with the use of eq (17).

between the oxide bias and internal quantum efficiency reflects the fact that the quantum yield for the 1337 type photodiode is greater than unity at $406 \mathrm{~nm}$. However, the fact that this discrepancy is not statistically significant at the one-sigma level shows that this conclusion cannot be drawn from the data presented here. The differences at the other wavelengths never exceed 0.0003 , and only fall outside the estimated uncertainties for the differences at $799.54 \mathrm{~nm}$.
The results shown in tables $5-7$ and figure 13 verify that the internal quantum-deficiency spectrum of multiple-reflection radiometers based on Hamamatsu 1337 photodiodes can be interpolated over the 440 to $860 \mathrm{~nm}$ spectral region from only two measured quantum deficiencies, one at each end of the region. This greatly reduces the number of measurements needed for high-accuracy spectral calibrations of these photodiodes and radiometers. Furthermore, the theoretical uncertainty analysis suggests that the uncertainties in the interpolated values will be less than or equal to the uncertainties in the measured values, at least as long as the latter are greater than \pm 0.0001 . The results in tables 5 to 7 and figure 13 do not contradict this idea, but the experimental data are not accurate enough to confirm it. On the other hand, the experimental data do confirm that an uncertainty of \pm 0.0003 is obtainable.

\section{Conclusion}

Part III of this series of papers has shown that Version 2 of the semiconductor-device modeling program PC-1D can be used to model the spectral shape of the internal quantum deficiency of 1337 type photodiodes with very small uncertainty over the $440 \mathrm{~nm}$ spectral region. The largest uncertainties are caused by uncertainties in the absorptioncoefficient spectrum of silicon, diode-to-diode variations in the shape of the front-region dopant distribution, and diode-to-diode variations in the position-independent rear-region minority-carrier lifetime. It was not possible to estimate the uncertainty caused by approximating the rear-region minority-carrier lifetime as being independent of position.

Simple formulae were derived that allow highaccuracy internal quantum-efficiency calibrations on 1337 type photodiodes to be interpolated from measured values at the ends of the 440 to $860 \mathrm{~nm}$ spectral region over the interior of that region. The uncertainties that can be obtained with these formulae are comparable to those that can be obtained with the highest accuracy measurements currently available.

\section{References}

[1] Geist, J., Appl. Opt. 18, 760 (1979).

[2] Geist, J., Zalcwski, E. F., and Schaefer, A. Appl. Opt. 19, 3795 (1980).

[3] Hoyt, C. C., Millcr, P. J., Foukal, P. V., and Zalewski, E. F., SPIE 1109, 236 (1989). 
[4] Zalewski, E. F., and Hoyt, C. C., Metrologia 28, 203 (1991).

[5] Geist, J., and Baltes, H., Appl. Opt. 28, 3929 (1989).

[6] Slotboom, J. W., and de Graaff, H. C., Solid-State Electron. 19, 857 (1976).

[7] Geist, J., Lowney, J. R., James, C. R., and Robinson, A. M., J. Appl. Phys. 70, 236 (1991).

[8] Ohkura, Y., Solid-State Electron. 33, 1581 (1990).

[9] Bennett, H. S., and Lowney, J. R., Solid-State Electron. 33, 675 (1990).

[10] Girisch, R. B. M., Mertens, R. P., and Verbeke, O. B., Solid-State Electron. 33, 85 (1990).

[11] Bennett, H. S., Solid-State Electron. 26, 1157 (1983).

[12] Geist, J., Migdall, A., and Baltes, H. P., Appl. Opt. 27, 3777 (1988).

[13] Weakliem, H. A., and Redfield, D., J. Appl. Phys. 50, 1491 (1979), and personal communication.

[14] Philipp, H. R., J. Appl. Phys. 43, 2835 (1972), and personal communication.

[15] Zalewski, E. F., and Duda, C., Appl. Opt. 22, 2867 (1983).

[16] Fox, N. P., and Martin, J. E., Appl. Opt. 29, 4686 (1990).

[17] Fox, N. P., Metrologia 28, 197 (1991), and personal communication.

About the authors: Jon Geist is a physicist in the NIST Semiconductor Electronics Division, and A. M. (Sandy) Robinson and C. R. (Bob) James are Professors of Electrical Engineering at the University of Alberta in Edmonton, Alberta, Canada. Prof. James is presently Vice-President (Research) at the University. 